\title{
Study of Tissue Printing Parameters for Generating Complex Tissue Constructs
}

\author{
G Navarro ${ }^{1 *}$, I Garcia ${ }^{1}$, PA Sundaram ${ }^{1}$ and N Diffoot-Carlo ${ }^{2}$ \\ ${ }^{1}$ Department of Mechanical Engineering, University of Puerto Rico, Mayagüez, 00680, Puerto Rico \\ ${ }^{2}$ Department of Biology, University of Puerto Rico, Mayagüez, 00680, Puerto Rico
}

*Corresponding author: Geoffrey Navarro Vale, B.S Recinto Universitario de Mayaguez Universidad de Puerto Rico, Isabela, PR Puerto Rico, Tel: 787955-0414; Email: geoffrey.navarro@upr.edu

Received date: July 22, 2016; Accepted date: August 12, 2016; Published date: August 20, 2016

Copyright: (C) 2016 Navarro G, et al. This is an open-access article distributed under the terms of the Creative Commons Attribution License, which permits unrestricted use, distribution, and reproduction in any medium, provided the original author and source are credited.

\begin{abstract}
A mixture of agarose, MEM IX and HeLa cells (dubbed Bio-Ink) was created to allow normal cell interaction with the scaffold material (agarose) before crosslinking as an initial step in 3D printing tissue. Bio-Ink was developed successfully as an in situ-scaffolding material for engineering biological structures. Bio-Ink has been further conditioned by adjusting agarose composition and gelling time to obtain optimal HeLa cell growth. After detailed study, the time range available for printing this material, before full crosslinking occurs, was determined to be about $300 \mathrm{~s}$, giving it attractive properties for 3D printing. Repeatable $10 \mathrm{~mm}$ thick prints were successful, although more system calibration is still needed to achieve more complex prints.
\end{abstract}

Keywords: 3D printer; Hydrogel; Agarose; HeLa

\section{Introduction}

There is a growing need for organ donors and substitutes to traditional drug testing. In the United States 18 people die daily waiting for an organ and more than 117,000 men, women and children await a life-saving organ transplant. Recently, 3D printing techniques are being employed to create complex biological structures with the long term goal of organ construction [1-5]. In this process, cells need to be seeded and cultured on printed scaffolds to generate tissue in a subsequent step. This process first identifies the composition and structure of the target tissue and tries to replicate it by incorporating the use of a bioprinter. The design tissue will then be constructed by a layer-by-layer technique in which a Bio-Ink is deposited on a surface being scaled up to build the targeted tissue. Another advantage of 3D printers is their ability to produce a high cell density print [2]. The printing of the scaffolds followed by subsequent cell seeding is a tedious process. Besides, it is complicated to incorporate physical characteristics in scaffolds to enable them to host cells which can proliferate successfully. Because $3 \mathrm{D}$ printers can create micro-scale structures, this integrated print technique can be incorporated in tissue printing to mimic the naturally occurring structures in the body, and enable better acceptance of the cells. In the current study, an attempt is made to print HeLa cells together with the agarose scaffold material and medium required for cell growth in one single step, therefore eliminating the need for a separate secondary process of seeding cells on a scaffold.

\section{Materials and Methods}

The selected material for this study was an agarose-based hydrogel which forms a 3 dimensional hydrophilic polymeric network. Hydrogels have a structure that is similar to the extracellular matrix (ECM) of many tissues, which will help with the cell integration $[1,6,7]$. Hydrogels also have the ability to homogenously encapsulate cells [1-6]. The natural biopolymer SeaKem agarose hydrogel was adapted as the in-situ scaffolding material. Agarose was added to
Minimum Essential Medium (MEM 1X), stirred and heated until boiling was achieved. Once sufficiently cool, HeLa cells were added into the mixture while still liquid. The Bio-Ink was prepared at different agarose concentrations $(1 \%, 1.25 \%$ and $1.4 \%)$, in order to determine optimum gelling temperature and structural rigidity. Data was obtained from a temperature vs. time plot for the duration of agarose crosslinking to determine the appropriate concentration which provided the best structural properties as well as printability. A $3 \mathrm{D}$ printer was chosen as the dispensing system due to its ability to produce complex micro-architectures which are needed in order to simulate the natural environment in which cells normally proliferate. The selected extrusion method for the 3D printer was originally to be a screw pump, due to its simple design and its ability to handle a wide range of fluids regardless of their viscosities [2]. However due to the inability to produce a constant stream flow of viscous material, the nozzle design was changed to a syringe pump. Vascularization, which occurs naturally in tissue to provide nutrients needed for cell growth, will be simulated by a rectilinear in-fill printing configuration pattern selected for the scaffold construct to enable the penetration of medium into the 3D structure to aid normal cell growth. HeLa cells were used for tissue printing due to their high resilience to harsh conditions. Cell proliferation and viability tests were conducted under the Bio-Ink preparation conditions as well as for the extrusion method.

\section{Results}

Preliminary findings for the agarose hydrogel include the correct mixture for the preparation of the Bio-Ink material and print parameters such as gelling temperature and its ability to maintain shape (rigidity). Figure 1 is a plot of temperature for the duration of hydrogel cooling while cross-linking to form the scaffold constructs. A mixture with $1.25 \%$ agarose concentration started crosslinking at 37 and reached full crosslinking at 32.7, while still retaining the desired structural integrity and rigidity, as shown in Figure 2. This is deemed as the best temperature range to incorporate cells while retaining cell viability during tissue printing as well as maintaining adequate printing parameters and feasible scaffold characteristics. In further experimentation, in separate culture flasks, cell proliferation was 
observed in the Bio-Ink after 6 days of incubation. Although confluency was expected (Figure 3A), the cells adopted a spherical morphology instead (Figure 3B). In order to determine if the viability of the cells was affected, some of the cells were retrieved from the printed Bio-Ink and cultured in a culture flask for further observation. These cells showed normal proliferation, similar to that seen in Figure $3 \mathrm{~A}$ with normal cell morphology and physiology after 6 days of incubation in the medium as seen in the image in Figure $3 \mathrm{C}$ validating the composition and physical characteristics of Bio-Ink. Initial prints using the $3 \mathrm{D}$ printer yielded low quality prints. Fine tuning and calibration of the printer software, mechanical interaction and heat transfer from the gel were implemented on initial prints to achieve better quality prints. The required mechanical properties of the gel were manipulated by adjusting the degree of cross-linked gel before extrusion. A simple agarose-only construct was realized (Figure 4A), although the 3D print model (Figure $4 \mathrm{~B}$ ) was further refined to print out a tissue construct from Bio-Ink including HeLa cells.

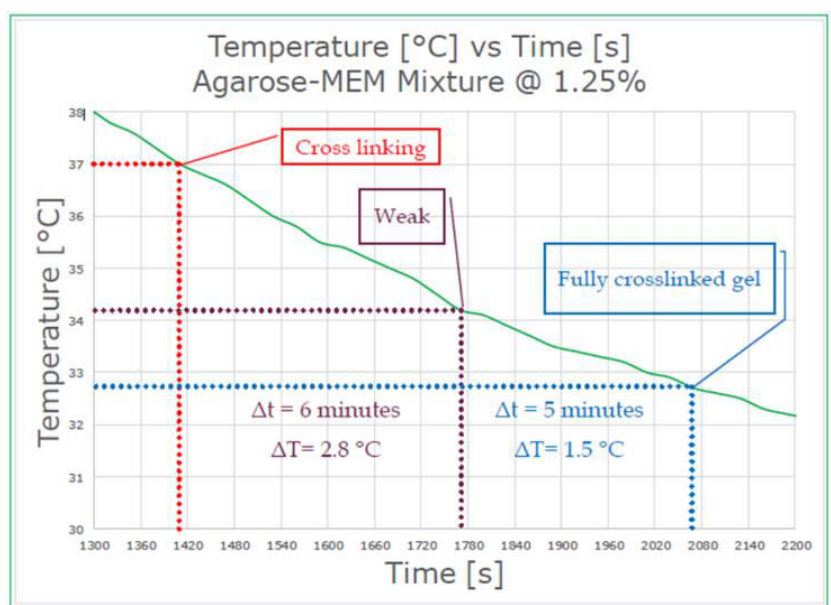

Figure 1: Temperature vs. time plot of hydrogel crosslinking at $1.25 \%$.

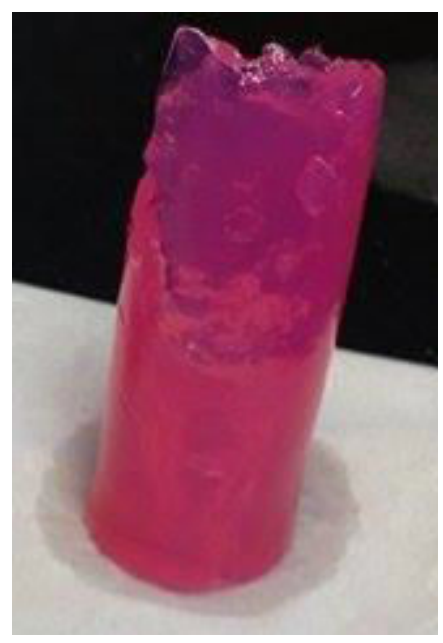

Figure 2: Image of stand-alone construct MEM IX and 1.25\% agarose mixture.

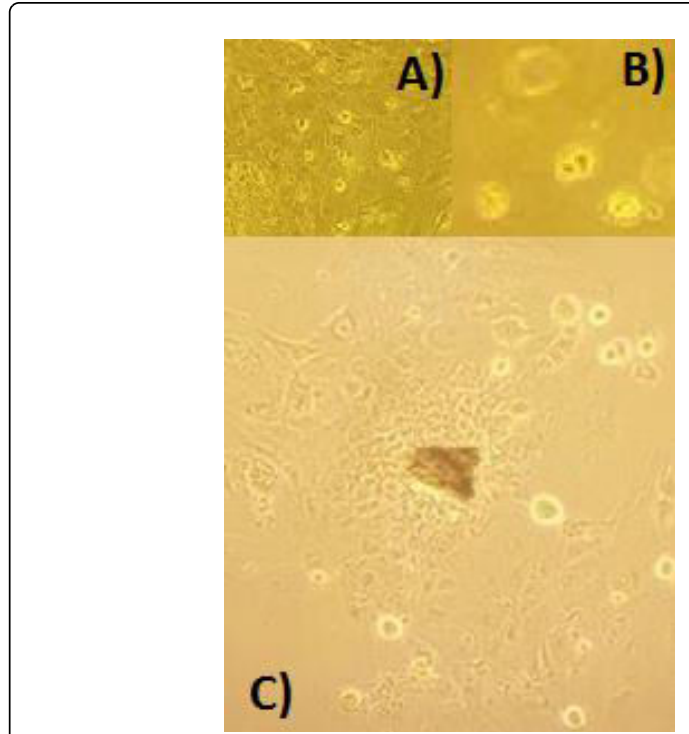

Figure 3: A) Normal confluent HeLa cell morphology. B) HeLa cell spherical morphology and cell proliferation in crosslinked agarose. C) HeLa cell morphology and proliferation in culture flask after being exposed to Bio-Ink preparation procedure.

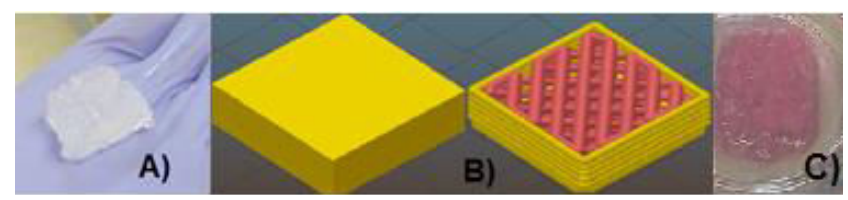

Figure 4: A) Agarose-only box print after some calibration B) 3D model to be printed C) Bio-Ink print.

Another example of a 3D model is shown in Figure 5A which was printed as an agarose-only ear (Figure $5 \mathrm{~B}$ ). Although, in the current set-up, the Bio-Ink stream diameter is $1.55 \mathrm{~mm}$, a better quality print of this ear using Bio-Ink can be achieved by reducing this syringe diameter.

\section{Discussion}

Agarose concentration of $1.25 \%$ for Bio-Ink material was selected due to its ability to maintain print shape with a starting gelling temperature of $37^{\circ} \mathrm{C}$ and full crosslinking at $32.7^{\circ} \mathrm{C}$ while being cooled. The syringe pump-like design was selected because it provided a better material stream during deposition that is essential for proper $3 \mathrm{D}$ construction. A nearly-constant stream of viscous material was achieved indicating that the $3 \mathrm{D}$ printer is capable of generating medium quality prints. Initial low quality prints served as a calibration baseline to improve future prints. Different mechanical and software parameters were fine-tuned, thus greatly improving the quality of the print as shown in Figure 4, were agarose-only prints and Bio-Ink print of a box of width and length of $20 \mathrm{~mm}$ and height of $10 \mathrm{~mm}$ were successfully printed repeatedly. These latest prints show that the printer is capable of handling the new material, and more importantly, better quality prints can be obtained. 
Citation: Navarro G, Garcia I, Sundaram PA, Carlo ND (2016) Study of Tissue Printing Parameters for Generating Complex Tissue Constructs. J Tissue Sci Eng 7: 175. doi:10.4172/2157-7552.1000175

Page 3 of 3

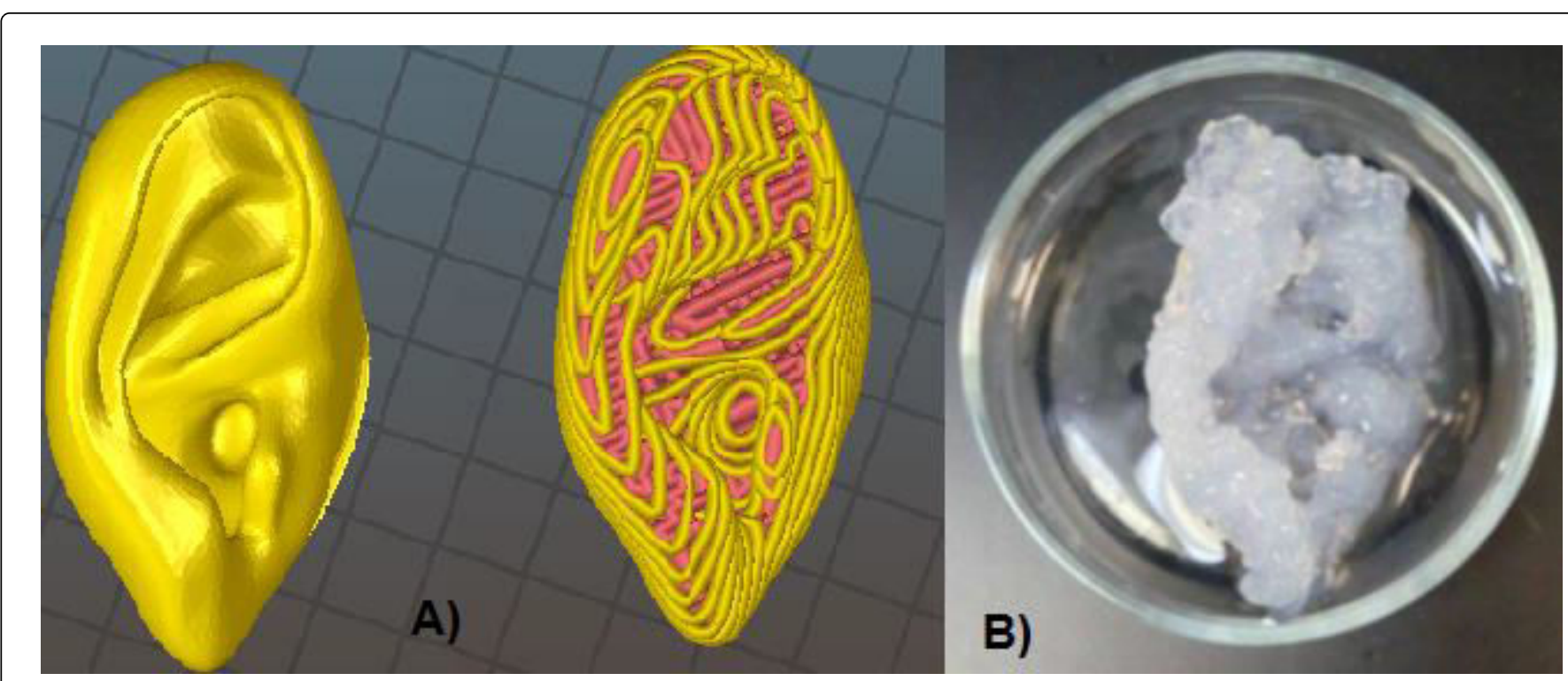

Figure 5: A) 3D model of ear to be printed B) Agarose-only ear print.

However when more complex structures like a human ear (Figure 5) were printed by this technique, the printer was unable to produce repeatable high quality prints and construct shape was affected. During the cell proliferation test, cell division was observed after 6 days of incubation, although the cells adopted a spherical morphology. However, during cell viability tests, normal cell function was observed with normal cell morphology after 6 days of incubation as seen in Figure 2. Also, no anomalies were observed on cell morphology or physiology after syringe extrusion. Currently, extrusion of Bio-Ink through a syringe pump-like mechanism to produced refined prints without producing any undesirable effect on the cells while maintaining good scaffold properties is being studied. Subsequently, agarose will be substituted with a biodegradable material such as chitosan and HeLa cells with human fetal osteoblasts. Tissue generated by the osteoblasts is expected to eventually replace chitosan in this process of engineering a solid tissue construct.

\section{Conclusion}

The Bio-Ink material was developed successfully as an in situ scaffolding material for engineering biological structures and deemed appropriate for proper cell growth environment due to proliferation of the HeLa cells in the Bio-Ink. The cells were able to proliferate and maintain viability inside the hydrogel. The Bio-Ink could be extruded with the syringe pump-like mechanism without producing any undesirable effect on the cells while maintaining good scaffold properties. Nevertheless, the complexity of the structures being printed are still relatively simple, and more calibration as well as more detailed prints through syringe diameter reduction is necessary. This proof-of- concept will be extended to fabricate structural elements required for replacement in the human body, after some more refinement of the printing parameters is achieved.

\section{Acknowledgement}

Howard Hughes Medical Institute funded this project under Grant No52007566.

\section{References}

1. Tan H, Marra KG (2010) Injectable, biodegradable hydrogels for tissue engineering applications. Materials 3: 1746-1767.

2. Murphy SV, Atala A (2014) 3D bioprinting of tissues and organs. Nat Biotechnol 32: 773-785.

3. Nguyen MK, Lee DS (2010) Injectable biodegradable hydrogels. Macromol Biosci 10:563-579.

4. Annabi N, Nichol JV, Zhong X, Ji C, Koshy S (2010) Controlling the porosity and microarchitecture of hydrogels for tissue engineering. Tissue Eng Part B Rev 16: 371-383.

5. Kang HW, Lee SJ, Ko IK, Kengla C, Yoo JJ, et al. (2016) A 3D bioprinting system to produce human-scale tissue constructs with structural integrity. Nat Biotechnol 34: 312-319.

6. Yanez M, De Maria C, Rincon J, Boland T (2011) Printable biodegradable hydrogel with self-crosslinking agent for wound dressing. Proc Int Conf on Digital Printing Technologies 632-635.

7. Billiet T, Vandenhaute M, Schelfhout J, Van Vlierberghe S, Dubruel P (2012) A review of trends and limitations in hydrogel-rapid prototyping for tissue engineering. Biomaterials 33: 6020-6041. 